\title{
The influence of floor type before and after 10 weeks of age on osteochondrosis in growing gilts ${ }^{1}$
}

\author{
D. B. de Koning, ${ }^{2,3}$ E. M. van Grevenhof, $\uparrow$ B. F. A. Laurenssen, * P. R. van Weeren, $\$$ W. Hazeleger,* \\ and B. Kemp*
}

\begin{abstract}
*Adaptation Physiology Group, Department of Animal Science, Wageningen University and Research Center, PO Box 338, 6700 AH Wageningen, The Netherlands; $\dagger$ Animal Breeding and Genomics Center, Department of Animal Science,

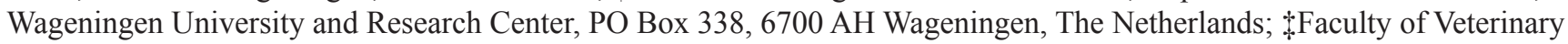
Medicine, Utrecht University, Department of Equine Sciences, PO Box 80.153, 3508 TD Utrecht, The Netherlands
\end{abstract}

\begin{abstract}
Osteochondrosis (OC) is a degenerative joint condition developing in a short time frame in young growing gilts that may cause lameness at an older age, affecting welfare and leading to premature culling of breeding sows. Causes of OC are multifactorial including both genetic and environmental factors. Floor type has been suggested to affect OC prevalence and effects might be age dependent during the rearing period. The aim of this study was to investigate possible agedependent effects of floor type, conventional concrete partially slatted versus wood shavings as deep bedding, on OC prevalence in gilts (Dutch Large White $\times$ Dutch Landrace) at slaughter (24 wk of age; 106.5 [14.7 SD] $\mathrm{kg}$ of BW). At weaning (4 wk of age; 6.9 [1.3 SD] kg of BW), 212 gilts were subjected to 1 of 4 flooring regimens. Gilts were either subjected to a conventional floor from weaning until slaughter (CC), wood shavings as bedding from weaning until slaughter (WW), a conventional floor from weaning until 10 wk of age after
\end{abstract}

which gilts were switched to wood shavings as bedding (CW), or wood shavings as bedding from weaning until 10 wk of age after which gilts were switched to a conventional floor (WC). After slaughter the elbow, hock, and knee joints were macroscopically examined for OC and scored on a 5 point scale where 0 indicates no OC and 4 indicates the severest form of OC. There was no significant difference $(P>0.4)$ between treatments on the overall OC prevalence for any joint assessed or at the animal level (all joints combined). At the animal level, however, gilts had greater odds to have OC scores 3 and 4 in the $\mathrm{CW}$ treatment (odds ratios $[\mathrm{OR}]=2.3$; $P=0.05)$, WC treatment $(\mathrm{OR}=2.6 ; P=0.02)$, and WW treatment $(\mathrm{OR}=3.7 ; P<0.001)$ compared with gilts in the $\mathrm{CC}$ treatment. The results indicate that there are no age-dependent effects of floor types on overall OC prevalence. However, wood shavings as bedding seems to increase the odds for severe OC and might affect animal welfare in the long term.

Key words: age, bedding, floor type, gilts, osteochondrosis

(C) 2014 American Society of Animal Science. All rights reserved.

J. Anim. Sci. 2014.92:3338-3347
doi:10.2527/jas2014-7902

\section{INTRODUCTION}

\footnotetext{
${ }^{1}$ This work was supported by the Science and Technology Foundation of the Netherlands Organization for Scientific Research (NWO-STW; grant number 11116), with co-financers Institute for Pig Genetics BV (IPG BV) and Product Board Animal Feed (PDV). The authors express their gratitude to the personnel of the experimental accommodation 'CARUS' for their care of the animals and assistance throughout the experiment.

${ }^{2}$ Corresponding author: danny.dekoning@wur.nl

${ }^{3}$ Present address: Adaptation Physiology Group, Department of Animal Sciences, Wageningen University and Research center, 6708 WD Wageningen, The Netherlands.

Received April 3, 2014.

Accepted May 20, 2014.
}

Osteochondrosis (OC) involves formation of necrotic growth cartilage in young gilts, which have been associated with leg weakness (Jørgensen and Andersen, 2000; de Koning et al., 2012) and premature culling (Yazdi et al., 2000). Prevalence of OC has been reported to range up to 80\% (Busch and Wachmann, 2011; van Grevenhof et al., 2011; de Koning et al., 2013) and culling due to leg weakness can range up to 30\% (Stein et al., 1990; Jørgensen and Sørensen, 1998). Breeding gilts last several parities and 
therefore it is important to reduce OC prevalence to avoid negative effects on welfare and longevity.

Floor types influence joint loading or the risk of traumatic events (such as slipping), which have been suggested to be associated with OC (Nakano and Aherne, 1988; Carlson et al., 1991; Ytrehus et al., 2004b,c). A conventional partially slatted floor as compared with deep litter has been reported to increase OC prevalence in fattening pigs (van Grevenhof et al., 2011), whereas others could not find an effect of floor type on OC prevalence (Jørgensen, 2003; Scott et al., 2006). Inconsistency in the effect of floor type on $\mathrm{OC}$ might be related with age dependency of OC development. Indications exist that a sensitive time period for porcine OC development lies around $10 \mathrm{wk}$ of age (Ytrehus et al., 2004a,b), which hence might imply age-dependent effects of floor types on OC prevalence as found earlier for dietary restriction (de Koning et al., 2013).

The aim of the study was to investigate age-dependent effects of floor type (conventionally partially slatted versus wood shavings) on OC prevalence in breeding gilts at $24 \mathrm{wk}$ of age, taking into account the abovementioned period of sensitivity for OC development. We hypothesized that a conventional floor increases OC prevalence as it is harder and more slippery. If floor type affects OC prevalence, it could be an environmental factor that might help in reducing OC prevalence in breeding gilts.

\section{MATERIALS AND METHODS}

\section{Ethical Note}

Osteochondrosis can cause joint surface irregularities possibly resulting in lameness that might affect welfare of the gilts. Assessments to detect serious impairments of welfare in the gilts were therefore performed daily. Severely lame or wounded gilts were taken out of the experiment and euthanized. The experiment and all measurements were approved by the Animal Welfare Committee of Wageningen University and Research center in compliance with Dutch law on animal experimentation.

\section{Animals}

The experiment was performed using 212 Topigs 20 (Dutch Large White $\times$ Dutch Landrace) gilts acquired from a commercial breeding company (TOPIGS, Veldhuizen Wehl, Wehl, the Netherlands). The Topigs 20 line represents a large part of the commercially kept sows in the Netherlands for the production of fattening pigs and previously it was found that OC prevalence within this line of animals ranges up to $60 \%$ (de Koning et al., 2013). The gilts were weaned at 27 (2.4 SD) d of age (6.9 [1.3 SD] kg of BW), after which they entered the experiment. Gilts had continuous access to various enrichment items (such as biting chains, burlap sacks, solid plastic balls, and/or rubber mats), which were changed every 2 to $3 \mathrm{~d}$. Gilts were given ad libitum access to water using drinking bowls and feed using a dry feeder unit.

\section{Treatments}

Gilts were assigned 1 of 4 treatments and divided to 32 pens $\left(8.37 \mathrm{~m}^{2}\right.$ surface area per pen $)$ of 6 to 7 individuals. Assignment to treatments was based on an equal distribution over pens of littermates and BW was measured at $19 \mathrm{~d}$ of age. Pens were distributed over 4 departments with an equal distribution of treatments per department. Gilts were housed either on a conventional floor consisting of $60 \%$ slatted floor (twisted metal bars) and $40 \%$ solid floor (epoxy-coated concrete) or on 25 to $50 \mathrm{~cm}$ deep bedding of wood shavings (approximately 3 to $5 \mathrm{~cm}$ large shavings). Wood shavings were gradually increased to approximately $50 \mathrm{~cm}$ bedding during the experiment. Wood shavings were distributed on the same floor type as the conventional floor except that the slatted area was covered with a $0.5 \mathrm{~cm}$ thick rubber mat so that wood shavings could not accumulate in the manure pan. The manure areas were removed as much as possible 3 to 4 times a week (approximately 10 to $15 \mathrm{~min}$ per pen) while gilts remained in their pens. After removal of manure, wood shavings were added to the existing layer of wood shavings to retain a full bedding of wood shavings. Daily inspections by the animal caretakers ensured that a full bedding was consistently available.

Treatments consisted of 4 flooring regimens administered to pens: a conventional floor from weaning until slaughter (CC), wood shavings as bedding from weaning until slaughter (WW), a conventional floor from weaning until $10 \mathrm{wk}$ of age after which gilts were switched to wood shavings as bedding $(\mathbf{C W})$, or wood shavings as bedding from weaning until $10 \mathrm{wk}$ of age after which gilts were switched to a conventional floor (WC). The switch in floor type was performed at $10 \mathrm{wk}$ of age (at an average of $74 \mathrm{~d}$ of age) so that the piglets received different floor types during the possible sensitive period for porcine OC development between 7 and 13 wk of age (Ytrehus et al., 2004a,b), during which OC can still be influenced.

Gilts received 4 successive diets according to their age and feed uptake. After weaning gilts received a pelleted weaning diet (11.0 MJ NE/kg, $170 \mathrm{~g} / \mathrm{kg} \mathrm{CP}, 12.5 \mathrm{~g} /$ $\mathrm{kg}$ ileal digestible lysine, $7.2 \mathrm{~g} / \mathrm{kg}$ calcium, and $5.5 \mathrm{~g} / \mathrm{kg}$ phosphorus). Gilts were switched at $40 \mathrm{~d}$ of age to a standard pelleted grower diet $(9.86 \mathrm{MJ} \mathrm{NE} / \mathrm{kg}, 165 \mathrm{~g} / \mathrm{kg} \mathrm{CP}$, $10 \mathrm{~g} / \mathrm{kg}$ ileal digestible lysine, $5.3 \mathrm{~g} / \mathrm{kg}$ calcium, and $5.3 \mathrm{~g} /$ $\mathrm{kg}$ phosphorus), at $70 \mathrm{~d}$ of age to a standard pelleted rearing diet $1(9.40 \mathrm{MJ} \mathrm{NE} / \mathrm{kg}, 157 \mathrm{~g} / \mathrm{kg} \mathrm{CP}, 8.4 \mathrm{~g} / \mathrm{kg}$ ileal digestible lysine, $8.5 \mathrm{~g} / \mathrm{kg}$ calcium, and $5.5 \mathrm{~g} / \mathrm{kg}$ phosphorus), and at $104 \mathrm{~d}$ of age to a standard pelleted rearing diet 
2 (9.24 MJ NE/kg, 140 g/kg CP, 6.9 g/kg ileal digestible lysine, $6.9 \mathrm{~g} / \mathrm{kg}$ calcium, and $5.1 \mathrm{~g} / \mathrm{kg}$ phosphorus).

\section{Behavioral Assessment}

As an indication of behavior performed that could influence results, behavioral activity was assessed at $8 \mathrm{wk}$ of age, $10 \mathrm{wk}$ of age ( 2 and $3 \mathrm{~d}$ after the switch in treatments), and 17 wk of age. Within each week, each pen was observed 4 times for 10 min continuously within 4 d. Observations were performed from 0800 to $1140 \mathrm{~h}$ in the morning and 1400 to $1740 \mathrm{~h}$ in the afternoon. The observation sessions were equally distributed for pens and treatments over days. Behavioral sampling was performed, focusing on the behaviors as described in Table 1. Within each 10 min observation session per pen, the frequency of a behavior performed per gilt within the pen was counted. Because of low frequencies of the behaviors observed, behaviors were combined per gilt in the categories play behavior and aggressive behavior and a category activity behavior. Play behavior consisted of the behaviors scamper, turn, flop, rolling, pushing, and gamboling. Aggressive behavior consisted of the behaviors chasing, fighting, and headbutting. Activity behavior consisted of the summation of play and aggressive behavior. Frequencies of behaviors were then summed per week as an indication of the number of behaviors performed within a week.

\section{Osteochondrosis Assessment}

Osteochondrosis assessment was performed after slaughter of the gilts at a local slaughterhouse. Gilts were slaughtered over a 1-wk period. One-half of the population with an equal distribution of treatments and pens was slaughtered within $2 \mathrm{~d}$ at an average of $166 \mathrm{~d}$ of age with an average BW of 105.6 (10.3 SD) kg. The other one-half of the population was slaughtered within $2 \mathrm{~d}$ at an average of $172 \mathrm{~d}$ of age with an average BW of 110.4 (10.1 SD) $\mathrm{kg}$. Carcasses were stored for $1 \mathrm{~d}$ at $4^{\circ} \mathrm{C}$ after which the legs from each gilt were collected by dissection through the shoulder and hip joints. The legs were then stored again at $4^{\circ} \mathrm{C}$ for a maximum of $2 \mathrm{~d}$ until dissection of the elbow, knee, and hock joints. Dissection of joints was performed within $1 \mathrm{~d}$ of the same slaughter week and occurred at a random order of gilts. After dissection of the joints, the joint surface was macroscopically assessed for presence and severity of OC using a 5-point grading scale from 0 to 4 (as described by van Weeren and Barneveld, 1999). The OC score 0 indicated no abnormalities, OC score 1 indicated flattening of the cartilage, OC score 2 indicated slight irregularities of the cartilage, OC score 3 indicated severe irregular cartilage, and OC score 4 indicated the severest form of $\mathrm{OC}$ with (partially) loose articular cartilage fragments and/or osteochondral cysts (for the locations as-
Table 1. Ethogram (taken with permission from Bolhuis et al., 2006)

\begin{tabular}{|c|c|}
\hline Behavior & Description \\
\hline \multicolumn{2}{|c|}{ Aggressive behavior } \\
\hline Chasing & Actively pursuing another pig \\
\hline Fighting & Mutual pushing or ramming, or lifting pen mate \\
\hline Headbutting & Ramming or pushing pen mate with head, without biting \\
\hline \multicolumn{2}{|l|}{ Play behavior } \\
\hline Scamper & A sequence of at least 2 forward hops in rapid succession \\
\hline Turn & Rapid turning around the body axis on the spot \\
\hline Flop & $\begin{array}{l}\text { A rapid drop from an upright position to sternal or lateral } \\
\text { recumbence }\end{array}$ \\
\hline Rolling & Lying on back and moving from side to side on the floor \\
\hline Pushing & Pushing the opponent with the head or shoulder \\
\hline Gamboling & $\begin{array}{l}\text { Running across the pen, occasionally accompanied by } \\
\text { nudging pen mate gently }\end{array}$ \\
\hline
\end{tabular}

sessed see de Koning et al. [2013]; for figures depicting OC lesions see van Grevenhof et al. [2011]). Osteochondrosis was scored by 1 veterinarian specialized in orthopedics, experienced in judging $\mathrm{OC}$, and unaware of the treatments.

\section{Statistics}

Behavioral Statistical Model. As the behaviors are frequencies and did not follow a normal distribution, the data was analyzed with a more appropriate distribution using a Poisson distribution with the default log link using PROC GLIMMIX in SAS 9.2 (SAS Inst. Inc., Cary, NC). The statistical model used to assess treatment effects on behavior is as follows:

$$
Y_{i j k l}=\mu+\text { Treat }_{i}+\operatorname{Dept}_{j}+\operatorname{Pen}_{k(i j)}+\varepsilon_{i j k l},
$$

in which $Y_{i j k l}$ represents the $i j k l$ th observation on behavior with a log link. The mean is represented by $\mu$. Treat $_{i}$ represents the fixed class effect of treatments administered $(i=\mathrm{CC}, \mathrm{CW}, \mathrm{WC}$, or $\mathrm{WW})$. Dept ${ }_{j}$ represents the fixed class effect of department in which gilts were housed ( $j=$ department $1,2,3$, or 4$)$ and is used to account for environmental variation. Pen $_{k(i j)}$ represents the random effect of the experimental unit pen $(k=$ pen 1 , $2, \ldots, 32)$ nested within treatment and department and is used to assess treatment effects on pen level. The random residual term from a Poisson distribution is represented by $\varepsilon_{i j k l}$. Results are displayed as the estimated least squares means and the SE on the log scale.

Osteochondrosis Affected Locations. The locations within joints on which no OC was found for all gilts were omitted from the analyses as these locations are uninformative. Bilateral symmetry of OC between joints of the bilateral homologues is indicated using Spearman rank correlations on the original OC scores using PROC CORR in SAS 9.2. 
Grouping of Osteochondrosis Scores. Osteochondrosis was scored on an ordinal scale of 0 to 4 . For each joint separately (joint level), the sufficient required number of observations of each combination of treatment and OC score could not be attained for application of ordinal logistic regression (Stokes et al., 2000) but was attained on the animal level (all joints combined). For the joint level, OC scores were grouped as a 0 and 1 variable to accommodate binary logistic regression, where 0 indicates no abnormalities (OC score 0 ) and 1 indicates an $\mathrm{OC}$ score greater than 0 . This procedure was also performed for the animal level. Additionally at the animal level, ordinal logistic regression was possible when grouping the OC scores to attain a sufficient number of observations between OC scores and treatments. For ordinal logistic regression, OC scores at the animal level were grouped into a variable with 3 categories: no OC (OC score 0 ), mild OC (OC scores 1 and 2), and severe OC (OC scores 3 and 4). To accommodate a repeated measurement analysis, the ordinal logistic regression method was approximated using a binary distribution and modeling the cumulative logits of ordinal logistic regression (for further details about this procedure and SAS code, see Stokes et al. [2000], p. 538).

Binary Logistic Regression. The OC scores were analyzed on pen level, which is the experimental unit. Osteochondrosis in joints was scored on the bilateral homologues and therefore observations within a gilt within a pen are not independent. A repeated measurement analysis was performed using PROC GLIMMIX in SAS 9.2. The statistical model used to assess treatment effects on OC prevalence is as follows:

$$
\begin{aligned}
\operatorname{Logit} P\left(y_{i j k l m}=1\right)= & \alpha+\operatorname{Treat}_{i}+\operatorname{Dept}_{j}+\operatorname{Pen}_{k(i j)}+ \\
& \operatorname{Gilt}(\text { Dam })_{l}+\varepsilon_{i j k l m},
\end{aligned}
$$

in which the model estimates the probability $(P)$ that the $i j k l m$ th observation of OC $(y)$ for a joint or at the animal level is 1 . Logit is the link function used to model the mixed linear regression analysis. The $\alpha$ component represents the estimate of the log odds of being affected with OC disregarding the independent variables (baseline odds [Kleinbaum and Klein, 2010]). Treat ${ }_{i}$ represents the fixed class effect of treatments administered $(i=\mathrm{CC}, \mathrm{CW}, \mathrm{WC}$, or WW). Dept ${ }_{j}$ represents the fixed class effect of department in which gilts were housed $(j=$ department $1,2,3$, or 4) and is used to account for environmental variation. $\mathrm{Pen}_{k(i j)}$ represents the random effect of the experimental unit pen $(k=$ pen $1,2, \ldots, 32)$ nested within treatment and department and is used to assess treatment effects on pen level. Gilt(Dam) $)_{l}$ represents the random term of gilts nested within dams from which the gilts originated. A repeated measurement analysis was performed on the binary OC scores from the different locations assessed for OC with gilts as the subject. The random residual term from a binary distribution is represented by $\varepsilon_{i j k l m}$. Addition of other variables such as slaughter weight, slaughter age, or birth weight did not significantly $(P>0.1)$ influence treatment effects on OC prevalence.

Ordinal Logistic Regression. The OC scores were analyzed on pen level, which is the experimental unit. As with the binary logistic regression model, a repeated measurement analysis was performed using PROC GLIMMIX in SAS 9.2. The statistical model used to assess treatment effects on OC prevalence is as follows:

$$
\begin{aligned}
& \operatorname{Logit} P\left(y_{i j k l m n}=1\right)=\alpha+\text { Treat }_{i}+\text { Dept }_{j}+\operatorname{Logtype}_{k}+ \\
& \left.(\text { Treat } \times \text { Logtype })_{i k}+\operatorname{Pen}_{l(i j)}+\text { Gilt(Dam }\right)_{m}+\varepsilon_{i j k l m n} \text {. }
\end{aligned}
$$

The terms in the ordinal logistic regression model remain similar as to the binary logistic regression model except for the addition of the cumulative logits modelled with ordinal logistic regression (Logtype ${ }_{k}=1,2$ ). In addition, an interaction is present between the cumulative logits and treatments $\left[(\text { Treat } \times \text { Logtype })_{i k}\right]$. This interaction allows the assessment of a difference between the prevalence of OC categories for the different treatments. Addition of other random variables such as slaughter weight, slaughter age, or birth weight did not significantly $(P>0.1)$ influence treatment effects on OC prevalence.

The prevalence of $\mathrm{OC}$ is presented on the ordinal scale and as the total number of OC affected joint locations and gilts. When significant treatment effects are present, odds ratios $(\mathbf{O R})$ are presented to indicate effect size.

\section{RESULTS}

Eleven out of the 212 gilts were removed before the end of the experiment due to health or welfare problems (3 gilts from the CC group, 3 gilts from the $\mathrm{CW}$ group, 3 gilts from the WC group, and 2 gilts from the WW group). The joints were assessed for OC status where possible after these animals were removed. Removals could not be attributed to treatment effects and were relatively equally distributed among treatments.

\section{Behavior}

The estimated least squares means from the statistical model concerning behaviors performed at 8,10 , and 17 wk of age are displayed in Table 2. No significant effects were present for aggressive behavior and activity behavior at all ages $(P>0.1)$. A significant difference was found for play behavior at $10 \mathrm{wk}$ of age $(P=$ $0.03)$ and a tendency for a difference at $17 \mathrm{wk}$ of age $(P=0.08)$. At $10 \mathrm{wk}$ of age, the gilts on wood shavings (CW and WW treatments) showed numerically more play behavior compared with gilts on conventional 
Table 2. Estimated least squares means $(\mathrm{SE})^{1}$ for aggressive behavior, play behavior, and activity behavior ${ }^{2}$ at 8,10 , and 17 wk of age per treatment ${ }^{3}$

\begin{tabular}{|c|c|c|c|c|c|c|c|c|c|}
\hline \multirow[b]{2}{*}{ Treatment } & \multicolumn{3}{|c|}{ Aggressive behavior } & \multicolumn{3}{|c|}{ Play behavior } & \multicolumn{3}{|c|}{ Activity behavior } \\
\hline & $8 \mathrm{wk}$ & $10 \mathrm{wk}$ & $17 \mathrm{wk}$ & $8 \mathrm{wk}$ & $10 \mathrm{wk}$ & $17 \mathrm{wk}$ & $8 \mathrm{wk}$ & $10 \mathrm{wk}$ & $17 \mathrm{wk}$ \\
\hline$\overline{\mathrm{CC}}$ & $0.96(0.16)$ & $0.66(0.20)$ & $0.77(0.19)$ & $1.80(0.34)$ & $0.61(0.35)^{\mathrm{ac}}$ & $-1.26(0.47)^{\mathrm{ac}}$ & $2.24(0.24)$ & $1.40(0.23)$ & $0.90(0.17)$ \\
\hline $\mathrm{CW}$ & $0.55(0.18)$ & $0.19(0.23)$ & $0.52(0.21)$ & $1.18(0.36)$ & $1.60(0.31)^{\mathrm{b}}$ & $-0.35(0.38)^{b c}$ & $1.70(0.26)$ & $1.87(0.21)$ & $0.89(0.17)$ \\
\hline WC & $1.07(0.15)$ & $0.67(0.20)$ & $0.66(0.20)$ & $2.12(0.33)$ & $0.04(0.40)^{\mathrm{c}}$ & $-1.47(0.51)^{\mathrm{a}}$ & $2.44(0.24)$ & $1.18(0.24)$ & $0.82(0.17)$ \\
\hline WW & $0.68(0.17)$ & $0.55(0.20)$ & $0.65(0.20)$ & $1.64(0.34)$ & $1.09(0.33)^{\mathrm{ab}}$ & $-0.05(0.37)^{\mathrm{b}}$ & $2.01(0.25)$ & $1.69(0.21)$ & $1.23(0.14)$ \\
\hline
\end{tabular}

${ }^{\mathrm{a}-\mathrm{c} W i t h i n ~ a ~ c o l u m n, ~ t h e ~ a m o u n t ~ b e h a v i o r ~ p e r f o r m e d ~ d i f f e r s ~ s i g n i f i c a n t l y ~ b e t w e e n ~ t r e a t m e n t s ~ i f ~ t h e r e ~ i s ~ n o ~ c o m m o n ~ s u p e r s c r i p t ~}(P<0.05)$.

${ }^{1}$ Estimated least squares means (SE) from the Poisson model on a natural log scale.

${ }^{2}$ Activity behavior is the summation of aggressive behavior and play behavior.

${ }^{3} \mathrm{CC}=$ a conventional floor from weaning until slaughter (51 gilts); $\mathrm{CW}=$ a conventional floor from weaning until 10 wk of age after which gilts were switched to wood shavings as bedding ( 50 gilts); $\mathrm{WC}=$ wood shavings as bedding from weaning until $10 \mathrm{wk}$ of age after which gilts were switched to a conventional floor (51 gilts); WW = wood shavings as bedding from weaning until slaughter (52 gilts).

flooring (CC and $\mathrm{WC}$ treatments). However, the significant differences were only found for the CW treatment compared with the CC and WC treatments and for the WC treatment compared with the $\mathrm{CW}$ and WW treatments. At 17 wk of age the gilts on wood shavings again displayed numerically more play behavior as compared with gilts on conventional flooring.

\section{Osteochondrosis Affected Locations}

Locations affected by OC in the elbow joint (total of 3 locations) were the medial humeral condyle (bilateral homologues) and the lateral humeral condyle (only in the left elbow joint), in the hock joint (total of 4 locations) were the lateral trochlea of the talus (bilateral homologues) and the medial trochlea of the talus (bilateral homologues), and in the knee joint (total of 2 locations) was the medial femoral condyle (bilateral homologues).

In the elbow joint the prevalence of OC scores greater than 0 was low (Table 3 ) and lesions were equally divided between the medial and lateral humeral condyles (data not shown). In the hock joint, the majority of OC scores greater than 0 were found on the medial trochlea of the talus $(93 \%$ of the total prevalence of OC scores greater than 0 ) of which $33 \%$ were scored as a 3 or a 4 (data not shown). Significant correlations $(P<0.001$ for all correlations) between bilateral homologues were found for the medial femoral condyle (correlation 0.39), the lateral trochlea of the talus (correlation 0.59), and the medial trochlea of the talus (correlation 0.52).

\section{Osteochondrosis Prevalence}

Descriptive results of the prevalence of the locations affected with OC within a joint and at the animal level are presented in Table 3. The hock joint had the greatest prevalence of $\mathrm{OC}$ affected locations and the greatest prevalence of OC scores 3 and 4. The elbow joint had the least prevalence of $\mathrm{OC}$ affected locations. The per- centage of OC scores 3 and 4 (more severe OC) of the overall prevalence of OC scores greater than 0 was $82 \%$ for the elbow joint, $31 \%$ for the hock joint, $57 \%$ for the knee joint, and $40 \%$ at the animal level.

The number of locations affected with OC does not necessarily represent the number of gilts affected with $\mathrm{OC}$, as a gilt may have bilateral symmetry of $\mathrm{OC}$ lesions at the joint level or have several joints affected with OC at the animal level. Descriptive results of the prevalence of gilts affected with their greatest OC score is displayed in Table 4. For example, if a gilt has OC scores on 2 joint locations with an OC score 1 and an OC score 4, then the greatest $\mathrm{OC}$ score is 4 and that animal is counted within the OC score 4 category of Table 4 . Again, the hock joint showed the greatest number of gilts affected with OC and the elbow joint showed the least number of gilts affected with OC. The percentage of gilts with OC scores 3 and 4 of the overall prevalence of gilts with $\mathrm{OC}$ scores greater than 0 was $82 \%$ for the elbow joint, $36 \%$ for the hock joint, $53 \%$ for the knee joint, and $48 \%$ at the animal level.

\section{Treatment Effects}

Descriptive results show that the prevalence of OC was greatest in the WC treatment except for the knee joint (Table 4). In the knee joint, OC prevalence was numerically greatest for the gilts in the WW treatment. The treatment or treatments with numerically the least number of gilts affected with $\mathrm{OC}$ in the elbow joint were the CC and WW treatments, in the hock joint the WW treatment, in the knee joint the $\mathrm{CW}$ treatment, and at the animal level the WW treatment. The WW treatment, therefore, seems to numerically be the treatment with the least number of gilts affected with OC. However, the WW treatment contained the greatest number of gilts affected with an OC score 3 and 4 at the animal level.

When OC was analyzed as a binary variable with binary logistic regression, no significant differences $(P>$ 0.4 ) were present between treatments for the different 
Table 3. Prevalence ( $n$ and \%) of osteochondrosis $(\mathrm{OC})^{1}$ on the locations within joints assessed per treatment and overall prevalence

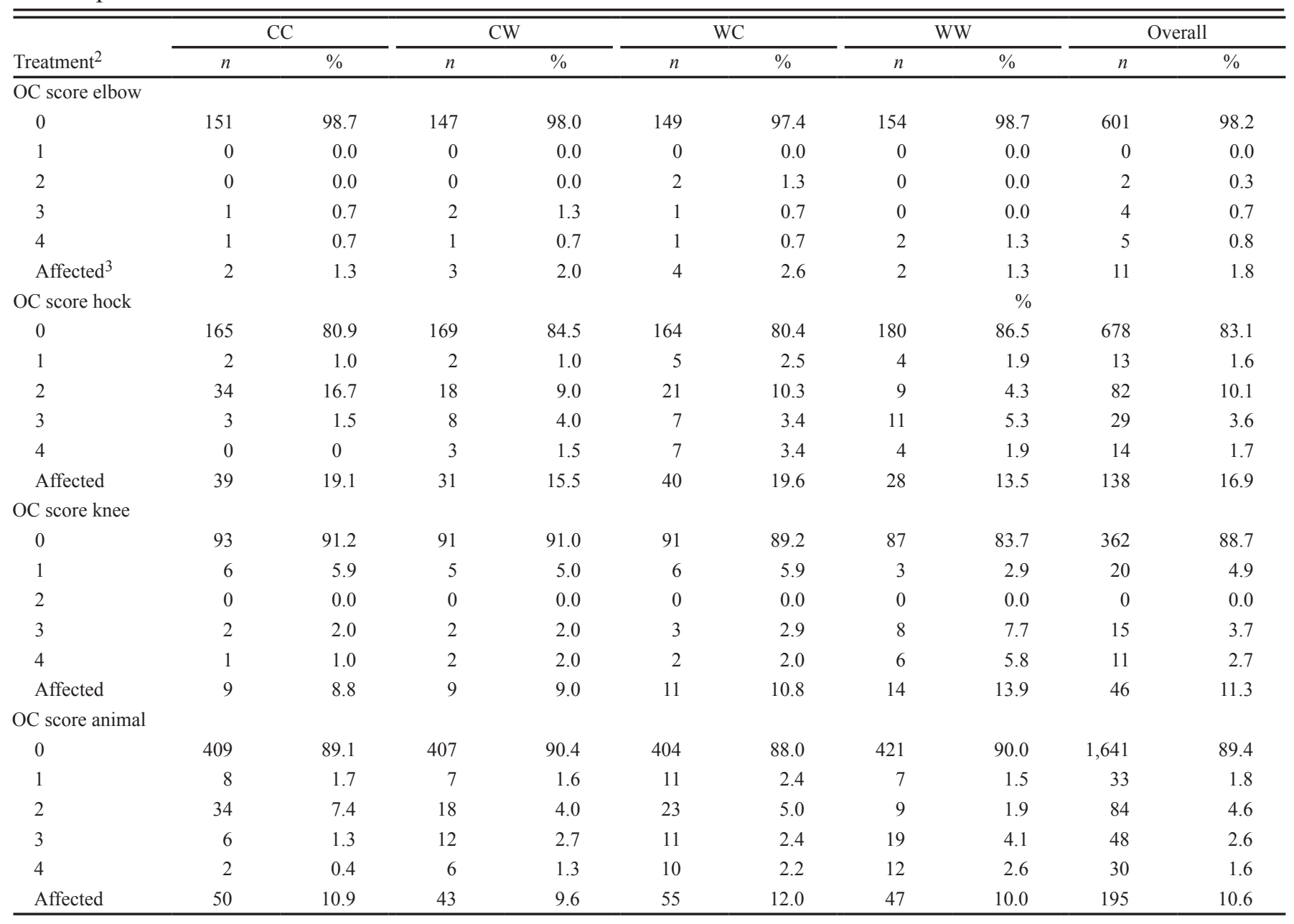

${ }^{1}$ Osteochondrosis was assessed on a 5-point scale were 0 indicates no OC and 4 indicates the severest form of OC for all the joint locations $(n$ and $\%$ of total n) assessed.

${ }^{2} \mathrm{CC}=$ a conventional floor from weaning until slaughter (51 gilts); $\mathrm{CW}=\mathrm{a}$ conventional floor from weaning until 10 wk of age after which gilts were switched to wood shavings as bedding ( 50 gilts); $\mathrm{WC}=$ wood shavings as bedding from weaning until $10 \mathrm{wk}$ of age after which gilts were switched to a conventional floor (51 gilts); WW = wood shavings as bedding from weaning until slaughter (52 gilts).

3"Affected" is the total number of locations with an OC score greater than 0.

joints or at the animal level. For the ordinal logistic regression model performed for the animal level, a significant effect was found for the interaction of logtype with treatment $(P<0.001)$. The interaction effect indicated that there was a greater odds to be affected within the category severe $\mathrm{OC}$ (OC scores 3 and 4 ) for gilts in the $\mathrm{CW}$ treatment $(\mathrm{OR}=2.3 ; P=0.05), \mathrm{WC}$ treatment $(\mathrm{OR}=$ $2.6 ; P=0.02)$, and $\mathrm{WW}$ treatment $(\mathrm{OR}=3.7 ; P<0.001)$ as compared with gilts in the $\mathrm{CC}$ treatment.

\section{DISCUSSION}

The aim of this study was to investigate age-dependent effects of floor type, conventional partially slatted versus wood shavings ( 25 to $50 \mathrm{~cm}$ of bedding), during the rearing period in breeding gilts on the prevalence of OC at 24 wk of age. Floor type might affect OC prev- alence and increase animal welfare. This would be in compliance with the EU directive 2008/120/EC stating that pig husbandry requires environmental enrichment of which bedding on floors would be a good candidate as supported by studies (Spoolder et al., 2000; Bolhuis et al., 2006; Averos et al., 2010). Results indicate that there does not seem to be a clear age-dependent effect of floor type on OC prevalence. However, the prevalence of severe OC lesions seems to be affected by floor type.

\section{Osteochondrosis Prevalence}

The overall prevalence of gilts affected with OC in the elbow and knee joint was relatively low (approximately 5 and $18 \%$, respectively). In a previous study that assessed the age-dependent effects of dietary restriction on OC prevalence in the same joint locations in the same 
Table 4. Prevalence ( $n$ and \%) of gilts of the total number of gilts within each treatment with a greatest osteochondrosis (OC) score $^{1}$ in the elbow joint, hock joint, knee joint, and at the animal level (all joints combined)

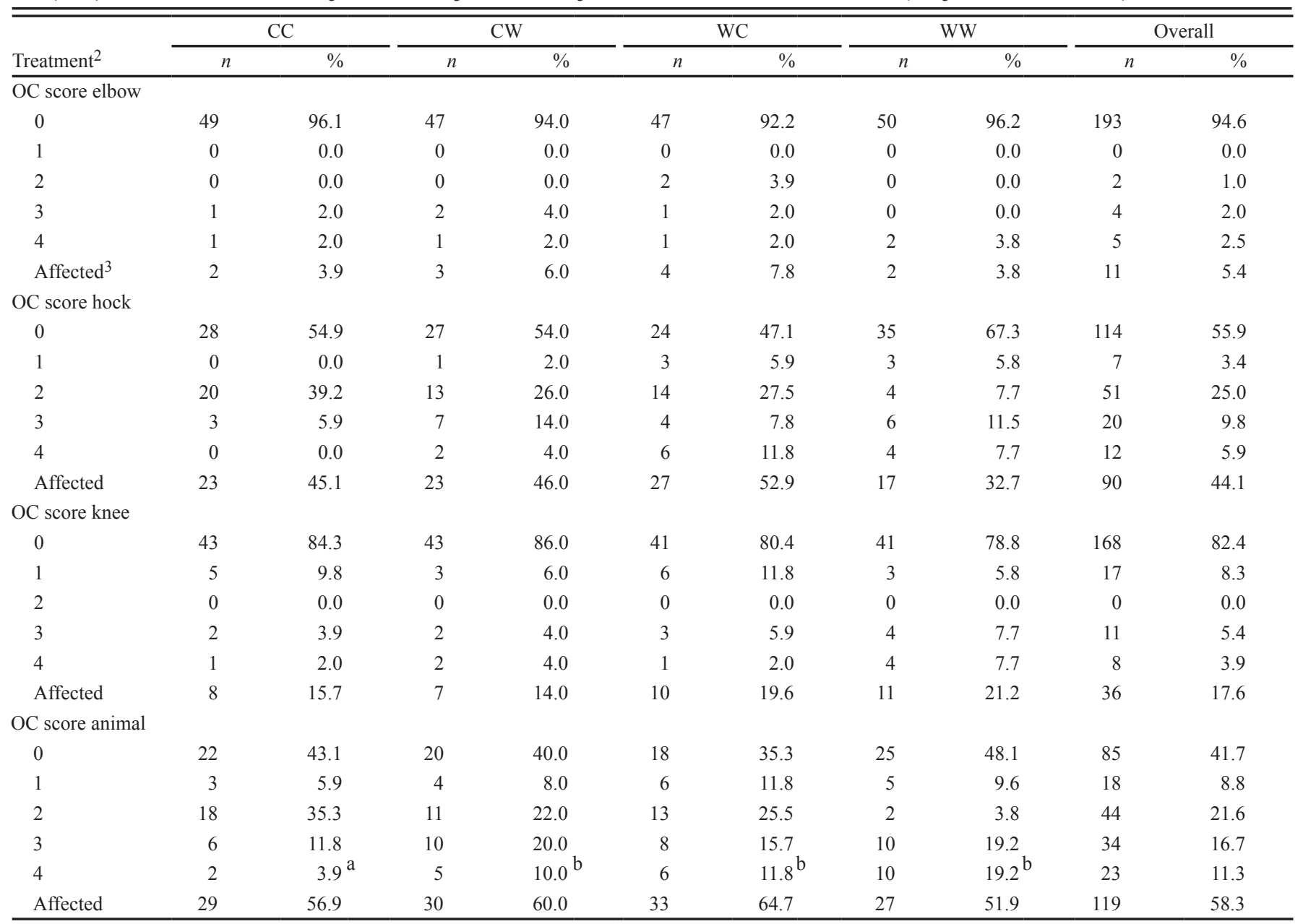

a,bSignificant differences $(P<0.05)$ between treatments were present for the severe OC lesions (OC score 3 and 4$)$ at the animal level. Within a row, the prevalence of OC differs significantly if there is no common letter $(P<0.05)$.

${ }^{1}$ Osteochondrosis was assessed on a 5 point scale were 0 indicates no OC and 4 indicates the severest form of OC.

${ }^{2} \mathrm{CC}=$ a conventional floor from weaning until slaughter $(n=51) ; \mathrm{CW}=$ a conventional floor from weaning until 10 wk of age after which gilts were switched to wood shavings as bedding $(n=50)$; $\mathrm{WC}=$ wood shavings as bedding from weaning until $10 \mathrm{wk}$ of age after which gilts were switched to a conventional floor $(n=51)$; WW = wood shavings as bedding from weaning until slaughter $(n=52)$.

3"Affected" is the total number of gilts with an OC score higher than 0.

genetic crossbred line of gilts (de Koning et al., 2013), overall OC prevalence in the elbow and knee joints were greater (approximately 18 and $41 \%$, respectively). The overall prevalence of gilts affected with OC in the hock joint, in contrast, was greater in the current study (approximately 44\%) as compared with our previous study (approximately 20\%). Similarly, the overall number of severe OC lesions (OC score 3 and 4) in the hock joint in the current study $(16 \%)$ was greater than in our previous study (3\%). However, the number of severe OC lesions in the hock joint is roughly equal between the $\mathrm{CC}$ treatment (approximately 6\%) of the current study and the comparable continuously fed ad libitum treatment from our previous study (approximately 8\%). The difference in OC prevalence is likely influenced by the difference in treatments imposed in this study and the previous study.
However, the prevalence of OC is known to vary between studies and seems to have a large range depending on the joint locations assessed. The prevalence of any form of OC (joint deformities indicative of OC) in any of the joints assessed might range up to 80\% (Lundeheim, 1987; Jørgensen, 1995, 2000; Jørgensen et al., 1995; Stern et al., 1995; Jørgensen and Andersen, 2000; Kadarmideen et al., 2004; Ytrehus et al., 2004c; Jørgensen and Nielsen, 2005; Luther et al., 2007; Busch and Wachmann, 2011; van Grevenhof et al., 2011). Reasons for this large range in OC prevalence between studies are likely various and might include factors such as breed and sex effects (Lundeheim, 1987; van der Wal et al., 1987), genetic predisposition towards OC development (Ytrehus et al., 2004c), or dietary restriction or type of housing applied (van Grevenhof et al., 2011; de Koning et al., 2013). 
Phenotypic bilateral symmetry of OC lesions as indicated by Spearman rank correlations was significant but low to moderate, ranging between 0.3 and 0.6. This is in concordance with a previous study performed by our group showing correlation coefficients in a similar range (de Koning et al., 2013). These results indicate that some phenotypic symmetry of OC lesions is to be expected but is not rigorously present and the joints from both sides are needed for proper OC assessment of an animal.

\section{Treatment Effects}

The different floor types used did not result in a significant difference in overall OC prevalence, nor were there indications of significant age-dependent differences in OC prevalence. Concerning overall OC prevalence, the WC and WW treatments had numerically the greatest and least numbers, respectively, of OC affected gilts in the elbow joint and hock joint and at the animal level. Concerning only severe lesions (OC score 3 and 4), gilts in the $\mathrm{CC}$ treatment had numerically consistently the least number of severe OC lesions, whereas gilts in WW treatment had the greatest number of gilts affected with severe OC lesions in the hock joint and knee joint and at the animal level. Significant effects were only present at the animal level, where the $\mathrm{CC}$ treatment contained significantly the least number of gilts affected with severe OC compared with the other treatments. There was no clear pattern of possible age-dependent effects of floor types. However, it seems that if gilts experience wood shavings as bedding either before or after $10 \mathrm{wk}$ of age or both, the number of severe OC lesions increase as compared with gilts kept continuously on a conventional concrete partially slatted floor. Previously, age-dependent effects of dietary restriction on OC prevalence were determined and a significant effect of the conditions that gilts experienced before and after $10 \mathrm{wk}$ of age on OC prevalence was found (de Koning et al., 2013). This is apparently not the case with floor types. However, in the current experiment, all gilts were fed ad libitum, which may have reduced contrasts between treatments.

To the knowledge of the authors, no study has been performed to determine whether the effect of floor type on OC prevalence is age dependent. In contrast to our results, van Grevenhof et al. (2011) showed that pigs housed on a conventional floor were more affected with OC than pigs housed on wood shavings as bedding. In agreement with our study, they also found that pigs housed on wood shavings as bedding had a greater prevalence of severe OC lesions. In addition, the difference of floor type found by van Grevenhof et al. (2011) was less present in pigs with restricted access to feed. This may indicate that effects of floor type can be influenced by the dietary restriction applied to pigs and may indicate that dietary restriction is a more important factor in reducing OC prevalence than floor type. Other studies were unable to report significant effects of floor types on OC prevalence (Jørgensen, 2003; Scott et al., 2006), which is in agreement to our results for overall OC prevalence.

\section{Possible Mechanism of Treatment Effects}

The results of this study did not support our hypothesis that a conventional floor would result in a greater prevalence of OC. Loading of joints and trauma to joints has been suggested to be associated with OC (Nakano and Aherne, 1988; Carlson et al., 1991; Ytrehus et al., 2004b,c). Impact loading on joints in normal motion would presumably be larger on concrete (hard) floors as compared with housing systems with wood shavings as bedding. Wood shavings as bedding would likely absorb more of the impact loading and thereby reduce the risk to develop OC. In addition, the conventional floor would be more slippery as compared with wood shavings as bedding. Conventional floors would, therefore, likely lead to more trauma to joints due to slipping of gilts and thereby increase the odds to develop OC. However, this study showed that gilts experiencing wood shavings as bedding before or after 10 wk of age or both have greater odds to be affected with severe OC than gilts housed continuously on a conventional floor. A possible speculative explanation to this might lie in the behavioral activity of the animals. In the current study, gilts on wood shavings showed more play behavior after $10 \mathrm{wk}$ of age as compared with gilts on conventional flooring. However, this was not reflected by the variable activity behavior, which was a summation of aggressive behavior and play behavior. Likely, the aggressive behavior affected the total frequency of activity behavior masking the effects of the frequency of play behavior. Other studies indicate that pigs housed on straw bedding or deep litter systems (somewhat comparable to wood shavings as bedding) are more active (Morgan et al., 1998; Lay et al., 2000; Bolhuis et al., 2005, 2006; Scott et al., 2006; van Grevenhof et al., 2011) and show more play behaviors (Lay et al., 2000; Bolhuis et al., 2005, 2006) as compared with pigs in conventional pens. A greater level of activity or play behavior might increase joint loading or increase the risk for trauma to the joints. Any mild OC lesions that would be present could then be aggravated by the joint loading and trauma, resulting in the progression to severe lesions. Conversely, gilts on a conventional floor might be less active and therefore less at risk to aggravate existing lesions. This might be in line with findings in horses where it has been shown that there may be an optimum between activity level and (intensity of) mechanical loading. In foals it has been shown that restriction of exercise and exercise in paddocks of a very large size or consisting of rough terrain or both increases the risk of development 
of OC lesions (Lepeule et al., 2013; Praud et al., 2013). This explanation of more activity leading to significantly more severe OC lesions, however, is not reflected by the observed behavior at $8 \mathrm{wk}$ of age before the treatment switch where there is not a clear difference in play behaviors performed. It is therefore likely that other, as of this point unknown, factors also have had an influence in the current results. In more practical terms it is important to notice that $\mathrm{OC}$ lesions within the classes 3 and 4 are more likely to lead to clinical effects or discomfort. Therefore, an increase in severe lesions is likely of more practical importance in terms of threats to welfare and longevity of sows that have to last several parities. In this manner, floor bedding could endanger animal welfare on the long term with regards to OC prevalence.

\section{Conclusion}

This study could not indicate age-dependent effects of the type of floor during the rearing period of gilts on the overall prevalence of OC. However, gilts kept on wood shavings as bedding either before or after $10 \mathrm{wk}$ of age or both had a greater odds to be affected with severe OC lesions as compared with gilts that were kept on a conventional floor from weaning until slaughter at $24 \mathrm{wk}$ of age. The exact biological mechanism to wood shavings as bedding increasing the odds of severe OC lesions remains elusive but may be related to a disturbance of the equilibrium between activity and mechanical loading of the joints. In practical terms, it seems that wood shavings as bedding at a relatively young age is to be avoided to reduce possible problems arising from $\mathrm{OC}$ at a later age (i.e., lameness).

\section{LITERATURE CITED}

Averos, X., L. Brossard, J. Y. Dourma, K. H. de Greef, H. L. Edge, S. A. Edwards, and M. C. Meunier-Salaun. 2010. A meta-analysis of the combined effect of housing and environmental enrichment characteristics on the behaviour and performance of pigs. Appl. Anim. Behav. Sci. 127:73-85.

Bolhuis, J. E., W. G. P. Schouten, J. W. Schrama, and V. M. Wiegant. 2005. Behavioural development of pigs with different coping characteristics in barren and substrate-enriched housing conditions. Appl. Anim. Behav. Sci. 93:213-228.

Bolhuis, J. E., W. G. P. Schouten, J. W. Schrama, and V. M. Wiegant. 2006. Effects of rearing and housing environment on behaviour and performance of pigs with different coping characteristics. Appl. Anim. Behav. Sci. 101:68-85.

Busch, M. E., and H. Wachmann. 2011. Osteochondrosis of the elbow joint in finishing pigs from three herds: Associations among different types of joint changes and between osteochondrosis and growth rate. Vet. J. 188:197-203.

Carlson, C. S., D. J. Meuten, and D. C. Richardson. 1991. Ischemic necrosis of cartilage in spontaneous and experimental lesions of ostochondrosis. J. Orthop. Res. 9:317-329. de Koning, D. B., E. M. van Grevenhof, B. F. A. Laurenssen, B. J. Ducro, H. C. M. Heuven, P. N. de Groot, W. Hazeleger, and B. Kemp. 2012. Associations between osteochondrosis and conformation and locomotive characteristics in pigs. J. Anim. Sci. 90:4752-4763.

de Koning, D. B., E. M. van Grevenhof, B. F. A. Laurenssen, P. R. van Weeren, W. Hazeleger, and B. Kemp. 2013. The influence of dietary restriction before and after 10 weeks of age on osteochondrosis in growing gilts. J. Anim. Sci. 91:5167-5176.

Jørgensen, B. 1995. Effect of different energy and protein levels on leg weakness and osteochondrosis in pigs. Livest. Prod. Sci. 41:171-181.

Jørgensen, B. 2000. Osteochondrosis/osteoarthrosis and claw disorders in sows, associated with leg weakness. Acta Vet. Scand. 41:123-138.

Jørgensen, B. 2003. Influence of floor type and stocking density on leg weakness, osteochondrosis and claw disorders in slaughter pigs. Anim. Sci. 77:439-449.

Jørgensen, B., and S. Andersen. 2000. Genetic parameters for osteochondrosis in Danish Landrace and Yorkshire boars and correlations with leg weakness and production traits. Anim. Sci. 71:427-434.

Jørgensen, B., J. Arnbjerg, and M. Aaslyng. 1995. Pathological and radiological investigations on osteochondrosis in pigs, associated with leg weakness. Zentralbl. Veterinarmed. A 42:489-504.

Jørgensen, B., and B. Nielsen. 2005. Genetic parameters for osteochondrosis traits in elbow joints of crossbred pigs and relationships with production traits. Anim. Sci. 81:319-324.

Jørgensen, B., and M. T. Sørensen. 1998. Different rearing intensities of gilts: II. Effects on subsequent leg weakness and longevity. Livest. Prod. Sci. 54:167-171.

Kadarmideen, H. N., D. Schwører, H. Ilahi, M. Malek, and A. Hofer. 2004. Genetics of osteochondral disease and its relationship with meat quality and quantity, growth, and feed conversion traits in pigs. J. Anim. Sci. 82:3118-3127.

Kleinbaum, D. G., and M. Klein. 2010. Chapter 1: Introduction to logistic regression. In: Logistic regression; A self learning text. 3rd ed. Springer Science+Business Media, New York, NY. p. 1-40.

Lay, D. C., Jr., M. F. Haussman, and M. J. Daniels. 2000. Hoop housing for feeder pigs offers a welfare-friendly environment compared to a nonbedded confinement system. J. Appl. Anim. Welf. Sci. 3:33-48.

Lepeule, J., N. Bareille, C. Robert, J. P. Valette, S. Jacquet, G. Blanchard, J. M. Denoix, and H. Seegers. 2013. Association of growth, feeding practices and exercise conditions with the severity of the osteoarticular status of limbs in French foals. Vet. J. 197:65-71.

Lundeheim, N. 1987. Genetic analysis of osteochondrosis and leg weakness in the Swedish pig progeny testing scheme. Acta Agric. Scand. 37:159-173.

Luther, H., D. Schworer, and A. Hofer. 2007. Heritabilities of osteochondral lesions and genetic correlations with production and exterior traits in station-tested pigs. Animal 1:1105-1111.

Morgan, C. A., L. A. Deans, A. B. Lawrence, and B. L. Nielsen. 1998. The effects of straw bedding on the feeding and social behaviour of growing pigs fed by means of single-space feeders. Appl. Anim. Behav. Sci. 58:23-33.

Nakano, T., and F. X. Aherne. 1988. Involvement of trauma in the pathogenesis of osteochondritis dissecans in swine. Can. J. Vet. Res. 52:154-155.

Praud, A., B. Dufour, C. Robert, J. P. Valette, J. M. Denoix, and N. Crevier-Denoix. 2013. Effects of management practices as risk factors for juvenile osteochondral conditions in 259 French yearlings. Vet. J. 197:72-76.

Scott, K., D. J. Chennells, F. M. Campbell, B. Hunt, D. Armstrong, L. Taylor, B. P. Gill, and S. A. Edwards. 2006. The welfare of finishing pigs in two contrasting housing systems: Fully-slatted versus straw-bedded accommodation. Livest. Sci. 103:104-115. 
Spoolder, H. A. M., S. A. Edwards, and S. Corning. 2000. Legislative methods for specifying stocking density and consequences for the welfare of finishing pigs. Livest. Prod. Sci. 64:167-173.

Stein, T. E., A. Dijkhuizen, S. D’Allaire, and R. S. Morris. 1990. Sow culling and mortality in commercial swine breeding herds. Prev. Vet. Med. 9:85-94.

Stern, S., N. Lundeheim, K. Johansson, and K. Andersson. 1995. Osteochondrosis and leg weakness in pigs selected for lean tissue growth rate. Livest. Prod. Sci. 44:45-52.

Stokes, M. E., C. S. Davis, and G. G. Koch. 2000. Generalized estimating equations. In: Categorical data analysis using the SAS system. 2nd ed. SAS Inst. Inc., Cary, NC. p. 471-550.

van der Wal, P. G., S. A. Goedegebuure, P. C. van der Valk, B. Engel, and G. van Essen. 1987. Leg weakness and osteochondrosis in pigs; Differences between the sexes of four breeds. Livest. Prod. Sci. 16:65-74.

van Grevenhof, E. M., S. Ott, W. Hazeleger, P. R. van Weeren, P. Bijma, and B. Kemp. 2011. The effects of housing system and feeding level on the joint-specific prevalence of osteochondrosis in fattening pigs. Livest. Sci. 135:53-61. van Weeren, P. R., and A. Barneveld. 1999. The effect of exercise on the distribution and manifestation of osteochondrotic lesions in the Warmblood foal. Equine Vet. J. Suppl. 31:16-25.

Yazdi, M. H., N. Lundeheim, L. Rydhmer, E. Ringmar-Cederberg, and K. Johansson. 2000. Survival of Swedish Landrace and Yorkshire sows in relation to osteochondrosis: A genetic study. Anim. Sci. 71:1-9.

Ytrehus, B., C. S. Carlson, N. Lundeheim, L. Mathisen, F. P. Reinholt, J. Teige, and S. Ekman. 2004a. Vascularisation and osteochondrosis of the epiphyseal growth cartilage of the distal femur in pigs- development with age, growth rate, weight and joint shape. Bone 34:454-465.

Ytrehus, B., S. Ekman, C. S. Carlson, J. Teige, and F. P. Reinholt. 2004b. Focal changes in blood supply during normal epiphyseal growth are central in the pathogenesis of osteochondrosis in pigs. Bone 35:1294-1306.

Ytrehus, B., E. Grindflek, J. Teige, E. Stubsjøen, T. Grøndalen, C. S. Carlson, and S. Ekman. 2004c. The effect of parentage on the prevalence, severity and location of lesions of osteochondrosis in swine. J. Vet. Med. A Physiol. Pathol. Clin. Med. 51:188-195. 